\title{
La Méditation du Poète
}

Ken Strickland

Nous sommes contents d'imprimer de temps en temps les meilleurs efforts de nos étudiants "undergraduatem et débutants. En voici un de ces essais, une explication du poème "Recueillement" de Baudelaire.

Le poème "Recueillement" de Charles Baudelaire a été écrit en 1'automne de 1866, une année avant que le poète ne meure au mois d'août 1867 . Le poème fait partie des Nouvelles Fleurs du mal qui a étê publiê en 1866.

Le poème est un sonnet français régulier écrit en vers alexandrins. Il se compose de deux rimes croisées identiques, suivies d'une rime plate et d'une autre rime croisée. Les rimes du premier quatrain sont suffisantes comme la deuxième rime du second quatrain. Les autres rimes sont riches. I1 y a aussi une alternance des rimes féminines et masculines dans les deux quatrains; la rime plate du premier tercet est féminine; dans la rime croisée suivante, une rime masculine alterne avec une rime féminine.

Dans ce poème, Baudelaire exprime une grande tristesse et la résignation résultante dont il ressent vers la fin de sa vie. Il se trouve dans une disposition de la soumission profonde et de la paix 
de l'esprit. Au milieu du néant, le poète peut encore avoir bon espoir, Sans doute, Baudelaire a choisi le titre "Recueillement" pour souligner la solennité de sa mêditation. Un sens presque religieux peut être tirê du mot; le poème lui-même se rapproche d'une prière avec sa langue musicale et harmonieuse.

Le poème s'ouvre sur un vers qui est à la fois réconfortant et surprenant. Le poète s'adresse à sa Douleur, mais il s.'exprime dans un style naĩf. Les mots dont il se sert sont ceux d'un monde enfantin--quotidiens, doux, cajoleurs et répétês. Le vers lui-même est presque saccadé, entremêlê de beaucoup de virgules. Par la langue on s'attendrait à ce que 1'auteur s'adresse à quelque chose de familier et d'intime, mais ce n'est pas ici exactement le cas car d'habitude on ne peut pas considérer le mot "douleur" comme cela. Il faut donc que cette Douleur de 1 'auteur soit regardée différemment qu'au sens propre, car il ne s'agit point d'une vraie souffrance insupportable. Peut-être qu'il s'agit de quelque remords ou de quelque amour non partagé. On ne peut que dire que cette Douleur est quelque souvenir qui a commencé dans le passé et qui a continué jusqu'au présent. En tout cas, 1'auteur lui-même est capable de nier cette Douleur en la projetant hors de lui. Il fait cela en la suppliant d'être sage et de se tenir plus tranquille.

La Douleur du poète est associée au soir dans le deuxième vers. Avec le commencement du soir vient le délai dont l'auteur se sert pour méditer sur ce qui s'était passé pendant la journée. Graduellement ses pensées s'étendent de plus en plus en arrière jusqu'à ce qu'il se souvienne de ce qui le hante toujours--cette Douleur non spécifiée. Le temps du verbe "réclamer" suggèłe aussi que ces opérations de son esprit arrivent assez souvent pour qu'il soit bien accoutumé à revenir à cette Douleur. I1 ne manifeste pas d'inquiétude. 
L'auteur personnifie le "Soir" en le rendant à peu près humain. La nuit tombe peu à peu, mais 1 'action paraît presque intentionnelle comme si le Soir était volontaire.

Dans le troisième vers le poète décrit plus précisement le décor où il se trouve. La ville qui est probablement Paris devient plus sombre. "Une atmosphère obscure enveloppe la ville." Au sens propre, ce $n^{\prime}$ est que le crépuscule approchant. Mais pour 1 'auteur, cela signifie quelque chose d'autre qui est bien évident dans le mot "obscure" et dans les vers précédents. Le Soir apporte sa Douleur; lui-même va être enveloppé par ses souvenirs et il ne peut pas les éviter. L'auteur reconnaît cela dans le quatrième vers, mais il porte l'idée plus loin. Quelques-uns d'entre les habitants de la ville reçoivent la paix, le contentement et le bonheur du soir. Pour eux, le soir est un temps pour être en repos. Mais pour les autres, le soir apporte le souci et $I^{\prime}$ inquiêtude comme il en apporte au poète.

Le ton du premier quatrain peut être divisé en deux parties. La première partie comprend les deux premiers vers. L'auteur se sert d'ordinaire de ces courtes phrases entrecoupées quand il s'adresse à sa Douleur. Il y a aussi beaucoup de sons comme dans les mots "sois" et "toi" qui pourraient suggérer la Douleur douce du poète. Dans les deux autres vers, le rythme devient plus régulier et harmonieux. Les Iiaisons du quatrième vers suggèrent 1 'approche graduelle du soir.

Le second quatrain fait contraste avec le début du poème. Au lieu de l'intimité du début, il y a des généralisations très abstraites. Maintenant 1 'auteur s'occupe de "la multitude vile / des mortels" de laquelle il a renversé 1 'ordre dans le cinquième vers. En faisant cela, il arrive à insister sur la mortalité des humains. Ils sont tous destinés à mourir. La mort devient donc très importante, car c'est la fin de la vie terrestre et le 
commencement d'une autre sorte de vie. On a $1^{\prime}$ impression que ces êtres mortels ne savent pas encore qu'il y a un rapport entre ce qu'ils font sur la terre et ce qui se passera dans $1^{\prime a u-d e l a ̀ . ~ P o u r ~}$ 1'auteur, ils s'appellent "la multitude vile"; ils perdent leur individualité et deviennent bas et ignobles.

Dans le sixième vers 1 'auteur crée une métaphore très curieuse. Le Plaisir devient une personne qui porte un fouet sous lequel se trouve la multitude vile. Ce paradoxe rappelle celui de la Douleur dars le premier quatrain. La Douleur possède des valeurs qui ne paraissent point si mauvaises; le poète y est bien accoutumé, C'est plutôt le Plaisir qui possède tous les attributs de la souffrance. L'apposition des mots "bourreau sans merci" explique plus la signification de ce Plaisir. Les êtres mortels sont complètement dominés par ce mâ̂tre; ils sont rẻduits en esclavage.

Cette idée se répète dans le septième vers. ". . la multitude vile / va cueillir des remords dans la fête servile. . .", c'est-à-dire que ces êtres sont en train de faire ce qu'ils regretteront à l'avenir selon toute probababłlité. A ce point s'élève un contraste entre les êtres qui cueillent des remords et le poète qui se recueille. On ne peut que se demander si ce n'est pas dans cette "fête servile" que le poète a trouvé ses remords.

Dans le huitième vers 1 'auteur recommence à s'adresser à sa Douleur dans de courtes phrases entrecoupées pareilles aux deux premiers vers du poème. Il trouve dans sa Douleur une sorte de con-tentement, une réconciliation avec le monde terres-tre. Il sait que par sa Douleur son âme sera sauvée dans le monde spirituel. Cela le rend plein d'espoir et de contentement.

Comme déjà mentionné ci-dlessus, le second quatrain se détache nettement sur le premier quatrain en utilisant 1 'image personnifiée du Plaisir. Ce ton plus solennel est aussi accompli par $1^{\prime}$ emploi 
de beaucoup de dentales dont le poète se sert pour évoquer la dureté de ce Plaisir. Cette strophe représente l'apogée du déchaînement des émotions du poète.

L'enjambement entre le huitième et le neuvième vers met en rejet les mots "loin d'eux." Cette brisure sépare effectivement de l'auteur le tumulte dans la ville. I1 s'éloigne physiquement des lieux de plaisir auxquels les êtres mortels assistent, car il trouve ces lieux dépjlaisants et car il ne s'intéresse plus à la volupté. Il est aussi lointain chronologiquement. C'est là autrefois qu'il a probablement fait ce qui le hante encore, ce qu'il ne peut jamais oublier.

Le poète continue à s'adresser à sa Douleur après la brisure dans le premier vers du premier tercet. Son état d'âme est devenu tranquille et contemplatif dans ces tercets. A cause de son isolement et son éloignement des êtres mortels, il n'a que ses remords pour se consoler. Il attire donc 1 'attention de sa Douleur sur quatre thèmes qu'il a transformés en personnifications. Il dit à sa Douleur de voir ces personnifications comme si elle avait des yeux et pouvait vraiment les voir.

La première personnification se trouve dans les premier et deuxième vers du premier tercet. C'est celle des "défuntes Années." E1les deviennent plusieurs vieilles femmes qui se perchent sur les nuages qui ont été transformés d'une manière concrète en "les balcons du ciel." Elles représentent sans doute les remords du poète qu'il a autrefois cueillis dans "1a fête servile." Ce souvenir de ce qui s'est passé en ce temps-là plane continuellement sur sa tête. Ainsi il se pourrait bien que le poète ne puisse jamais l'oublier. En outre, ces vieilles femmes portent des "robes surannées." Cela suggère qu'elles représentent aussi le passé et la fuite du temps.

Dans le troisième vers du premier tercet la 
personnification du Regret se présente d'une manière qui sort de $1^{\prime}$ ordinaire. D'habitude on tiendrait le regret pour un chagrin ou pour un dêsappointement, Ici, cela peut être vrai, mais ce Regret sourit aussi. I1 faut donc que ce Regret soit pareil à la Douleur du poète. C'est quelque chose de rêconfortant, car le poète sait que s'il regrette sincèrement ce qu'il a fait dans le monde temporel, il fera son salut dans le monde spirituel.

Le Regret revêt un sens primordial dans la première partie de ce même yers quand le poète le qualifie d'une force surgissante du fond des eaux, Ceci évoque l'image de la première vie animale qui s'élève de l'océan dans le temps préhistorique. Ainsi ce Regret représente quelque chose d'éternel et d'omniprésent. Chacun va ressentir du regret un jour ou 1 'autre de son vivant. Ce Regret représente bien plus le reflet des "défuntes Années" sur la surface des eaux, ce qui explique aussi l'êmergence du fond des eaux. Un reflet donne souvent 1 "impression de surgir du fond de 1 'eau, comme le regret du fond du souvenir. De cette façon, le passê ('les dêfuntes Années") est reflêtê par son souvenir (les eaux), ce qui le mène au Regret, Le poète en a conscience maintenant et on peut aussi dire que les êtres mortels qui prennent part actuellement à "la fête servile" ressentiront probablement du même sentiment à 1 'avenir.

La troisième personnification est amplifiée dans le premier vers du second tercet. "Le Soleil moribond" représente le jour qui est presque accompli. Cette image qui est créée par l'adjectif "moriborı" est très êvocatrice, Les rayons lumineux baissent comme s'ils étaient en train de mourir. Mais ce coucher du soleil se passe tranquillement et paisiblement; il n'y a pas jde dérangement. Simplement, le jour passant "s'endort sous une arche," ce qui évoque l'image d'un tableau encadré. L'arche du pont sert à séparer d'une manière concrète le 
jour et les expériences du poète. De cette façon ce jour devient une partie du passé et se sépare à jamais du prêsent.

Les deux derniers vers du poème développent la dernière personnification qui est celle de la Nuit. Le passage du premier tercet au second tercet est montré par la transformation des "robes surannées" en "un long linceul traînant à 1'Orient." I1 commence à faire plus sombre, et la Nuit qui tire son origine de l'est s'approche peu à peu, Ici, le mot "Orient" donne une image exotique et mystérieuse à la Nuit. L'auteur fait un rapprochement entre les deux images du Soleil et de la Nuit en transformant 1a Nuit en "un long Iinceul," Ce linceul va couvrir complètement le Soleil qui est en train de mourir.

Le poète en revient à une dernière phrase courte et entrecoupée quand il s'adresse encore une fois directement à sa Douleur dans le dernier vers du poème. Auparavant il a demandé à sa Douleur de voir "se pencher les défuntes Annēes," de voir "surgir. - .le Regret souriant" et de voir "le Soleil moribond s'endormir." A ce point, il fait appel à un autre sens de la Douleur personnifiée. Il la prie d'entendre "la douce Nuit qui marche," La Douleur est devenue "ma chère," ce qui souligne encore une fois que le poète considère sa Douleur comme quelque chose de familier et d'intime. L'itmage de la Nuit s'achève par 1'adjectif "douce" et par la proposition relative "qui marche." La Nuit approche graduellement du poète en apportant avec elle le réconfort des ténèbres. C'est le temps qui est employé par le poète à méditer sur ce qui s'était passé de son vivant. Il médite sur ses remords et sur ses regrets, ce qui fait naître toujours sa Douleur.

Les images des deux tercets sont très fortes et évocatrices en elles-mêmes. Mais le poète les rend plus frappantes en se servant de beaucoup de sons divers. Les sons vocaliques des mots "loin 
d'eux" suggèrent $1 \mathrm{a}$ distance entre le poète et la multitude. Les consonnes des mots "balcons du ciel" expriment la soliditê des nuages qui supportent "les défuntes Années." Les mots "surgir du fond des eaux" évoquent le mouvement ascendant du Regret. Les sons vocaliques des mots "un long linceul traînant à l'Orient" insistent sur la longueur et la largeur de la Nuit approchante. La suite Iambique dans le dernier vers évoque par sa monotonie et son mouvement la marche assourdie de la Nuit.

Dans ce poème, Baudelaire réussit à décrire son état d'âme vers la fin de sa vie. I1 se trouve loin de tous les autres; il s'est éloigné d'eux à la fois physiquement et chronologiquement. Il médite paisiblement sur sa vie, mais il ne s'en contente pas. Il a des remords qui occasionnent sa Douleur. I1 espère pourtant faire son salut dans le monde spirituel $s^{\prime}$ il peut sincèrement regretter ses actions dans le monde temporel, Le poème monte à un crescendo dans les deux quatrains et puis finit par presque chuchoter les deux tercets, ce qui ressemble à une prière, L'aboutissement des efforts de Baudelaire est frappant et touchant.

The University of Kansas

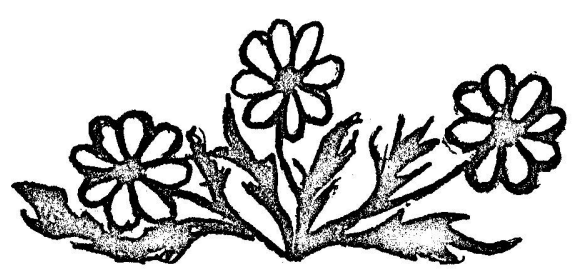

